\title{
Extended Law of Corresponding States Applied to Solvent Isotope Effect on a Globular Protein
}

\author{
Saskia Bucciarelli, ${ }^{\dagger}$ Najet Mahmoudi, ${ }^{*}{ }^{\dagger}$, Lucía Casal-Dujat, $^{\dagger}$ Marie Jéhannin, ${ }^{\dagger}$ Corinne Jud, ${ }^{\ddagger}$ \\ and Anna Stradner* ${ }^{*} \dagger$ \\ ${ }^{\dagger}$ Physical Chemistry, Department of Chemistry, Lund University, SE-22100 Lund, Sweden \\ ${ }^{\ddagger}$ Adolphe Merkle Institute (AMI), University of Fribourg, CH-1700 Fribourg, Switzerland \\ Supporting Information
}

ABSTRACT: Investigating proteins with techniques such as NMR or neutron scattering frequently requires the partial or complete substitution of $\mathrm{D}_{2} \mathrm{O}$ for $\mathrm{H}_{2} \mathrm{O}$ as a solvent, often tacitly assuming that such a solvent substitution does not significantly alter the properties of the protein. Here, we report a systematic investigation of the solvent isotope effect on the phase diagram of the lens protein $\gamma \mathrm{B}$-crystallin in aqueous solution as a model system exhibiting liquid-liquid phase separation. We demonstrate that the observed strong variation of the critical temperature $T_{c}$ can be described by the extended law of corresponding states for all $\mathrm{H}_{2} \mathrm{O} / \mathrm{D}_{2} \mathrm{O}$ ratios, where scaling of the temperature by $T_{c}$ or the reduced second virial coefficient accurately reproduces the binodal, spinodal, and osmotic compressibility. These findings highlight the impact of $\mathrm{H}_{2} \mathrm{O} / \mathrm{D}_{2} \mathrm{O}$ substitution on $\gamma \mathrm{B}$-crystallin properties and warrant further investigations into the universality of this phenomenon and its underlying mechanisms.

Limit of stability (spinodal)

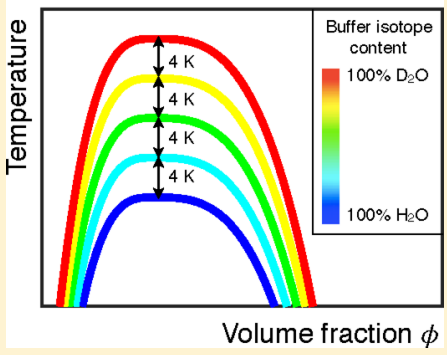

ince its first production by Lewis and MacDonald in 1933 $\checkmark$ and despite its low natural availability (at one part in about 6500 parts of light water), ${ }^{1}$ heavy water $\left(\mathrm{D}_{2} \mathrm{O}\right)$ and its biological effects have been investigated extensively. ${ }^{2}$ Studies included, for example, the cytotoxic and cytostatic activities of $\mathrm{D}_{2} \mathrm{O}$ against human cancer cells, ${ }^{3}$ its contradictory effects on cellular secretion, ${ }^{4}$ and the absence of retinal toxicity when $\mathrm{D}_{2} \mathrm{O}$ is used in bovine and human retina perfusion studies. ${ }^{5}$ Furthermore, solvent isotope effects in protein studies have contributed to a deeper understanding of protein structural stability. Indeed, secondary structure studies of disparate proteins have shown that the folded state is mostly stabilized in $\mathrm{D}_{2} \mathrm{O} .^{6-11}$ Additionally, $\mathrm{D}_{2} \mathrm{O}$ was shown to increase protein stability against thermal denaturation. ${ }^{12-14}$

Some experimental techniques, such as nuclear magnetic resonance (NMR), spectroscopic techniques, ${ }^{15,16}$ and neutron scattering, ${ }^{17,18}$ commonly used to investigate protein internal and solution structure and dynamics often rely on solvent isotope substitution, partially or completely replacing $\mathrm{H}_{2} \mathrm{O}$ by $\mathrm{D}_{2} \mathrm{O}$. In coherent neutron scattering methods, deuterium is used instead of hydrogen in order to decrease the background arising from the incoherent scattering of the latter or to perform contrast variation studies on multicomponent systems. ${ }^{19}$ In infrared spectroscopy, ${ }^{15}$ vibrational circular dichroism $^{20}$ and $\mathrm{NMR}^{21}$ the signal from water absorption bands interferes considerably with that from protein molecules, thus making the use of a deuterated solvent necessary. In interpreting the results of such measurements, the effects of solvent isotope on protein colloidal stability are frequently left out and it is often assumed that proteins have essentially the same solution structure in $\mathrm{H}_{2} \mathrm{O}$ and $\mathrm{D}_{2} \mathrm{O}$. Earlier work, however, has shown that lysozyme, a globular protein with a short-range attractive interaction potential, is less soluble in $\mathrm{D}_{2} \mathrm{O}$ than in $\mathrm{H}_{2} \mathrm{O},{ }^{22,23}$ which is linked to enhanced proteinprotein attractions in $\mathrm{D}_{2} \mathrm{O} .^{24}$ Stradner et al. ${ }^{25}$ found that using $\mathrm{D}_{2} \mathrm{O}$ as solvent for lysozyme strongly affects its colloidal stability. Additionally, the effect of isotope substitution on solvent properties, and consequently on interactions and phase behavior in other colloidal systems such as polymer solutions and surfactant systems, is also well established. ${ }^{19}$ This clearly calls for an in-depth investigation of the effect of solvent isotope content on the phase behavior and interaction potential governing solutions of globular proteins, in order to help clarify to which extent results obtained in solvents with different isotope content can be compared and used to draw conclusions on living systems, such as cells or the eye lens, which contain only $\mathrm{H}_{2} \mathrm{O}$. We have therefore performed a systematic study of the phase behavior and the solution structure of the globular protein $\gamma \mathrm{B}$-crystallin, one of the major components of the eye lens, in aqueous solutions of varying deuterium content.

$\gamma \mathrm{B}$-crystallin, the smallest of the eye lens proteins with a radius of about $1.8 \mathrm{~nm}$ is commonly used as a model system for colloidal particles interacting via a short-range attractive interaction potential. ${ }^{26}$ It displays a complex phase behavior that combines liquid-liquid phase separation and dynamical arrest $^{27-29}$ with an arrest line that extends into the unstable region below the spinodal, a feature previously also observed in lysozyme. $^{30}$ The equilibrium phase diagram of the latter was

Received: March 14, 2016

Accepted: April 14, 2016

Published: April 14, 2016 
shown to follow the extended law of corresponding states (ELCS) of Noro and Frenkel, ${ }^{31}$ whereby the critical point and the connected coexistence curve at various ionic strengths are rescaled using the reduced second virial coefficient $b_{2}$, defined as the ratio of the second virial coefficient $B_{2}$ and $B_{2}^{\mathrm{HS}}=2 \pi \sigma^{3} / 3$, the second virial coefficient of a solution of hard spheres with the same diameter $\sigma$ as the protein. This demonstrates that the critical point and the coexistence curve of lysozyme depend on the integral features of its interaction potential and not on the details of the latter. ${ }^{32,33}$ Moreover, Gripon et al. ${ }^{22}$ reported that in a salt-concentration range of $0.3-0.6 \mathrm{M} \mathrm{NaCl}$, increasing the concentration of $\mathrm{NaCl}$ by $0.1 \mathrm{M}$ was found to have the same effect on the solubility of lysozyme as substituting $\mathrm{H}_{2} \mathrm{O}$ by $\mathrm{D}_{2} \mathrm{O}{ }^{22}$ We may thus speculate that the ELCS applies not only to globular proteins in solutions with different ionic strength but also to solvents with varying hydrogen isotope content.

In this study, we investigate the effect of the gradual substitution of $\mathrm{D}_{2} \mathrm{O}$ for $\mathrm{H}_{2} \mathrm{O}$ in $\gamma \mathrm{B}$-crystallin solutions on their phase behavior and the strength of their interaction potential. We show, through a combination of transmission measurements, static light scattering (SLS), and small-angle X-ray scattering (SAXS), that increasing the $\mathrm{D}_{2} \mathrm{O}$ content of the solvent has a large effect on both and that the validity of the ELCS can indeed be extended to $\mathrm{H}_{2} \mathrm{O} / \mathrm{D}_{2} \mathrm{O}$ substitution.

We located the metastable binodal (or coexistence curve for liquid-liquid phase separation) and the spinodal of $\gamma \mathrm{B}$ crystallin solutions in five different solvents with varying $\mathrm{H}_{2} \mathrm{O} / \mathrm{D}_{2} \mathrm{O}$ content (cf. Table 1, cf. Supporting Information

Table 1. Mixing Ratios of $\mathrm{H}_{2} \mathrm{O}$ and $\mathrm{D}_{2} \mathrm{O}$ Used in This Study and Corresponding Data Symbols Used in the Graphs

\begin{tabular}{cccl} 
ratio & vol $\% \mathrm{H}_{2} \mathrm{O}$ & vol $\% \mathrm{D}_{2} \mathrm{O}$ & data symbol \\
$100 / 0$ & 100 & 0 & green $\triangle$ \\
$75 / 25$ & 75 & 25 & pink $\nabla$ \\
$50 / 50$ & 50 & 50 & blue $\square$ \\
$25 / 75$ & 25 & 75 & orange $\diamond$ \\
$0 / 100$ & 0 & 100 & purple $\bigcirc$ \\
\hline
\end{tabular}

(SI) for details). The resulting data is shown in Figure 1a and b. The critical temperature $T_{\mathrm{c}}$ is clearly strongly affected by the amount of deuterium in the solution, as summarized in the inset of Figure 1. Fitting the left $\left(\phi<\phi_{\mathrm{c}}\right)$ and right $\left(\phi>\phi_{\mathrm{c}}\right)$ branches of all binodals and spinodals with

$$
T=T_{c}\left[1-A\left(\frac{\left|\phi-\phi_{c}\right|}{\phi_{c}}\right)^{1 / \beta}\right]
$$

with individual fitting parameters $A$ and a critical exponent $\beta=$ $0.33,{ }^{29}$ we find that $\phi_{c}$ on the other hand remains unaffected by $\mathrm{D}_{2} \mathrm{O}$ and that the shape of the curves does not depend on solvent isotope content. In fact, the same fitting parameters $A_{\mathrm{b}, \text { left }}=(0.06 \pm 0.02)$ and $A_{\mathrm{b}, \text { right }}=(0.02 \pm 0.01)$ reproduce all the binodals and the same $A_{\mathrm{s}, \text { left }}=(0.39 \pm 0.08)$ and $A_{\mathrm{s}, \text { right }}=$ $(0.08 \pm 0.04)$ reproduce all the spinodals (cf. Figure la and $b)$. This becomes even more apparent when scaling the temperature by $T_{\mathcal{O}}$ which causes the curves to superimpose, as shown in Figure 1c and d, reminiscent of the results reported by Gibaud et al. ${ }^{32}$ and by Platten et al. ${ }^{33}$ for lysozyme in solutions with varying ionic strength. These authors have shown that under these conditions, the Noro-Frenkel ELCS applies and that the reduced second virial coefficient $b_{2}$ can be used to predict the phase behavior of the protein. To test whether the isotope effect on the phase behavior of $\gamma \mathrm{B}$-crystallin can also be predicted based on the ELCS, we have experimentally determined $B_{2}$ from SLS on dilute solutions in 100/0, 50/50, and $0 / 100$ solvent, following the method described by Gibaud et al. $^{32}$ (cf. SI for details). The resulting temperature-dependent $b_{2}$ values are shown in Figure 2a. At the critical temperature $T_{\mathcal{c}}$ we find $b_{2}\left(T_{c}\right)=-2.7 \pm 0.5$ for all three solutions under investigation. When scaled to $T_{\mathcal{C}}$ all $b_{2}$ indeed fall onto one master curve, as shown in Figure $2 \mathrm{~b}$. We discuss the following results in light of a simple square-well (SW) potential

$$
U_{\mathrm{SW}}(r)=\left\{\begin{array}{cc}
\infty & r<\sigma \\
-\varepsilon & \sigma \leq r \leq \Lambda \sigma \\
0 & r>\Lambda \sigma
\end{array}\right.
$$

describing the interactions as a combination of a hard core repulsion and a short-range attraction of strength (or well depth) $\varepsilon$ and range $\Lambda$. In accordance with earlier results, ${ }^{29,30}$ we take $\Lambda=1.20$, that is, a range of the attractive interaction potential corresponding to $20 \%$ of the protein diameter. $b_{2}$ is then given by

$$
b_{2}\left(T^{*}\right)=1-\left(\Lambda^{3}-1\right)\left(\exp \frac{1}{T^{*}}-1\right)
$$

where $T^{*}=k_{\mathrm{B}} T / \varepsilon \cdot{ }^{33}$ Following the approach of Platten et al., ${ }^{33}$ we assume a linear dependence of the SW depth on temperature and fit $b_{2}\left(T / T_{c}\right)$ with

$$
b_{2}\left(\frac{T}{T_{c}}\right)=1-\left(\Lambda^{3}-1\right)\left[\exp \left(\frac{a T / T_{c}+b}{T / T_{c}}\right)-1\right]
$$

The fitting parameters $a$ and $b$ were found to be $a=-3.3 \pm 0.5$ and $b=5.0 \pm 0.5$. Combining eqs 3 and 4 , we obtain the depth of the SW as a function of $T / T_{c}$

$$
\frac{\varepsilon}{k_{\mathrm{B}} T}=\frac{a T / T_{\mathrm{c}}+b}{T / T_{\mathrm{c}}}
$$

as shown in Figure 2c. Using eq 4, temperatures can universally be converted to $b_{2}$ and vice versa, as demonstrated in Figure $1 \mathrm{c}-\mathrm{f}$, where the binodals and spinodals obtained in all solvents superimpose when plotted in the $T / T_{c}-\phi$ plane, as well as in the $b_{2}-\phi$ plane. The ELCS proposed by Noro and Frenkel, ${ }^{31}$ thus, can indeed accurately reproduce the phase diagram of this globular protein in solutions with different hydrogen isotope content.

To further investigate the validity of the ELCS, we now focus on $S^{-1}(0)$, the inverse static structure factor in the forward direction, related to the osmotic compressibility $\kappa_{T}$ of the solution. We extracted $S^{-1}(0)$ from SAXS data, following the method described in an earlier publication. ${ }^{29}$ Examples of static structure factors $S(q)$ for samples close to $\phi_{c}$ in $100 / 0,50 / 50$, and $0 / 100$ solvents are given in Figure $3 a-c$, respectively. The critical (low- $q$ ) parts of the structure factors over a wide range of volume fractions were fitted with the $\mathrm{OZ}$ equation

$$
S(q, T, \phi)=\frac{S_{\text {crit }}(0)}{1+q^{2} \xi_{\mathrm{s}}^{2}}+S_{\text {non-crit }}
$$

which is the sum of a strongly temperature-dependent critical component $\left(S_{\text {crit }}(0) /\left(1+q^{2} \xi_{s}^{2}\right)\right)$, associated with the shortrange attractions, and a temperature-independent noncritical background ( $\left.S_{\text {non-crit }}\right)$, assumed to be $q$-independent in the low$q$ range $\left(q \ll q^{*}\right)$ considered here. ${ }^{34}$ As shown in Figure $4 c$, the 

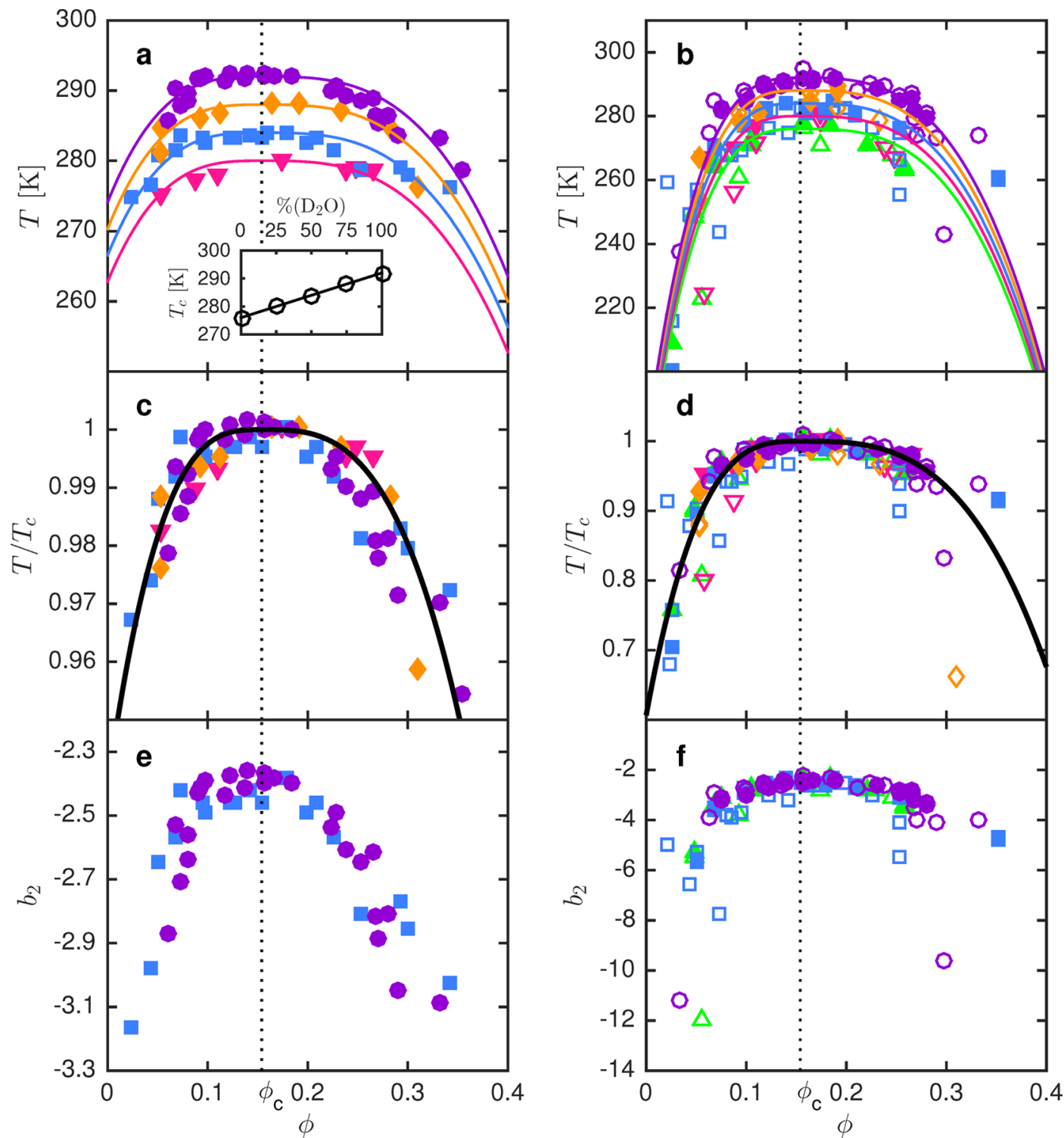

Figure 1. Binodal (left) and spinodal (right) of $\gamma$ B-crystallin solutions in solvents with varying hydrogen isotope content. (a) and (b) Unscaled data. (c) and (d) Data from (a) and (b) scaled by $T_{\mathrm{c}}$ (e) and (f) Binodals and spinodals in the $b_{2}-\phi$ plane, where $b_{2}$ is the reduced second virial coefficient. The solid lines show the fits to eq 1 and the dotted line marks $\phi_{c}$. Right: filled symbols correspond to $T_{\text {sp }}$ from Ornstein-Zernike $(\mathrm{OZ})$ fits to SAXS structure factors (eq 6) and open symbols correspond to $T_{\mathrm{sp}}$ from SLS. Inset: $T_{\mathrm{c}}$ of $\gamma \mathrm{B}$-crystallin solutions as a function of solvent $\mathrm{D}_{2} \mathrm{O}$ content.
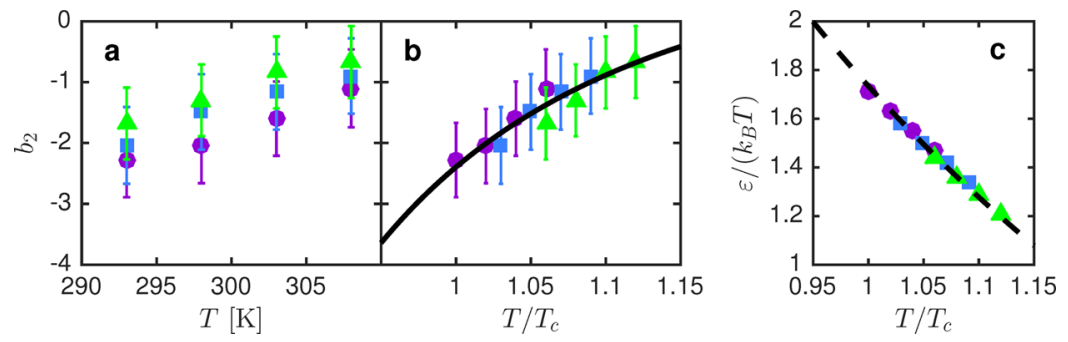

Figure 2. Reduced second virial coefficient $b_{2}$ of $\gamma \mathrm{B}$-crystallin in solvents with varying deuterium content, (a) as a function of temperature and (b) scaled by $T_{\mathrm{c}}$. The solid black line shows the global fit (eq 4) to all data sets. (c) Well depth $\varepsilon /\left(k_{\mathrm{B}} T\right)=1 / T^{*}$ of the SW potential as a function of reduced temperature. The dashed black line corresponds to eq 5 .

latter follows the Carnahan-Starling (CS) prediction for $S(0)$ of hard spheres, that is, it is associated with the hard sphere interactions of the proteins. Moreover, all data points superimpose without further scaling, confirming that the hard core repulsion of the protein remains unaffected by solvent isotope substitution. $S_{\text {crit }}(0)=S_{0} \tau^{-\gamma}$, shown in Figure $4 \mathrm{~b}$, is the critical component of the static structure factor in the forward direction and $\xi_{s}=\xi_{s, 0} \tau^{-\nu}$ is the static correlation length that 


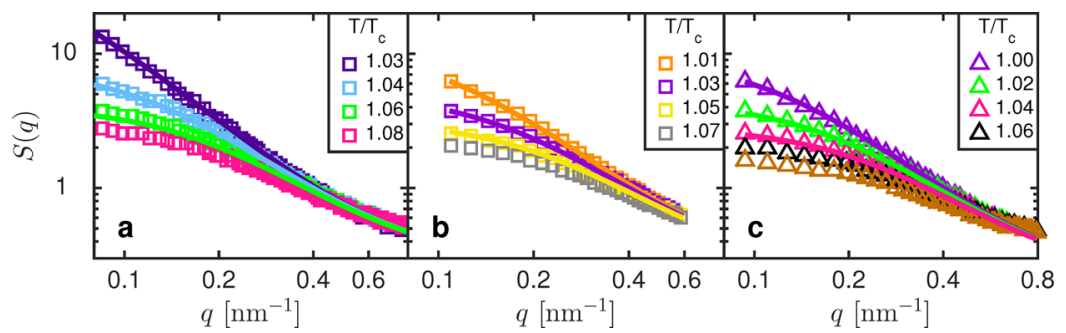

Figure 3. Critical part of the experimentally determined static structure factors $S(q)$ (data symbols) at $\phi=0.16$ (close to $\left.\phi_{c}\right)$ in (a) 100/0, (b) 50/ 50 , and (c) $0 / 100$ solvents, together with the $\mathrm{OZ}$ fits to the critical parts of the static structure factors close to criticality (eq 6, lines) at varying $T / T_{\mathrm{c}}$.

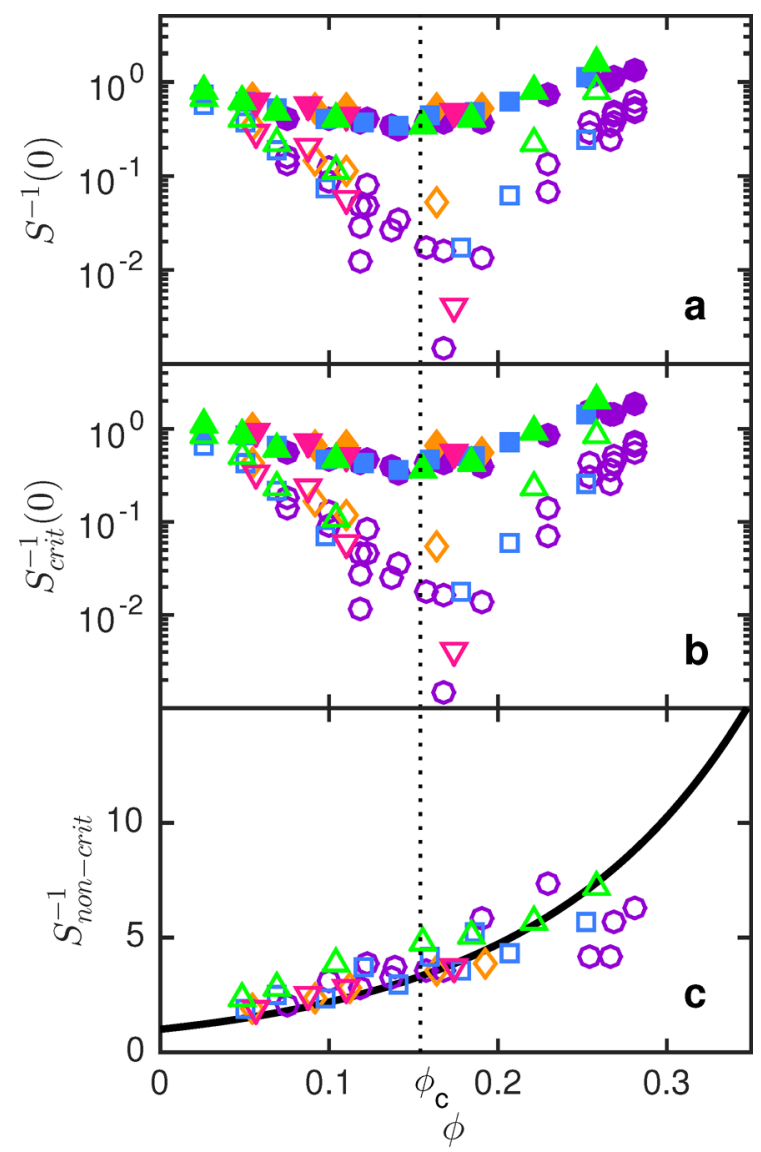

Figure 4. (a) $S^{-1}(0)$ obtained from SAXS (from OZ fits) for different solvent isotope content, scaled by $T_{c}$ and (b) critical component $S_{\text {crit }}^{-1}(0)$, scaled by $T_{\mathrm{c}}$ at $T / T_{\mathrm{c}} \approx 1.06$ (filled symbols) and $T / T_{\mathrm{c}} \approx 1$ (open symbols). (c) Noncritical, $T$-independent background of $S(0)$. Also shown is the CS prediction for hard spheres (solid black line). The dotted line marks $\phi_{c}$. Cf. Figure S2 in the SI for 3D plots of (a) and (b) containing the full data set over a large range of $T / T_{\mathrm{c}}$.

measures the characteristic length scale of density fluctuations caused by critical phenomena. $\nu=0.63$ and $\gamma=1.24$ are the critical exponents from the Ising universality class ${ }^{35,36}$ and $\tau=$ $\left(T-T_{\mathrm{sp}}\right) / T_{\mathrm{sp}}$ is the reduced temperature. This allows us to extract the spinodal temperature $T_{\mathrm{sp}}$ from OZ fits to $S(q)$ at each $\phi$ using $S_{0}, \xi_{\mathrm{s}, 0}, T_{\mathrm{sp}}$, and $S_{\text {non-crit }}$ as fitting parameters and simultaneously fitting the structure factors at all measured $T$ close to criticality with eq 6 (lines in Figure 3). We then find an average value of $\xi_{\mathrm{s}, 0}=(7.01 \pm 0.74) \mathrm{nm}$ for the five solvents, independently of $\phi$, which is in agreement with the value of $(7.27 \pm 0.71) \mathrm{nm}$ found in pure $\mathrm{D}_{2} \mathrm{O}$ solvent. ${ }^{29}$ Moreover, the thus obtained $T_{\mathrm{sp}}$ values (filled symbols on the right-hand side of Figure 1) closely follow the spinodal obtained from SLS (open symbols). We now focus on low $q$ values and thus length scales significantly larger than the protein size, that is, on the total forward structure factor $S(0)=S_{\text {crit }}(0)+S_{\text {non-crit }}$ shown in Figure $4 \mathrm{a}$ for the five hydrogen isotope contents investigated at two different temperatures scaled by $T_{c} \cdot S^{-1}(0)$ exhibits a minimum around $\phi_{\mathrm{c}}$ for a given temperature, due to the critical component, which also exhibits a minimum at the same $\phi$ (cf. Figure $4 \mathrm{~b}$ ). More importantly, the $T_{\mathrm{c}}$-scaling causes $S^{-1}(0)$ and $S_{\text {crit }}^{-1}(0)$ corresponding to the different solvents to superimpose, similarly to the phase diagram. The ELCS, using $T / T_{c}$ (or $b_{2}$ ) as a scaling parameter thus also applies to the osmotic compressibility.

In summary, the phase boundaries for liquid-liquid phase separation and the osmotic compressibility of aqueous solutions of the short-range attractive globular protein $\gamma \mathrm{B}$-crystallin scale with the reduced second virial coefficient $b_{2}$, quantifying protein-protein interactions, through the extended law of corresponding states (ELCS). This thermodynamic scaling confirms the applicability of the ELCS to the equilibrium properties of colloids with short-range attractions ${ }^{32,37}$ and provides an extension of its predictive power to systems with varying hydrogen isotope content. Using a simple square-well (SW) fluid analysis, we moreover show that the well depth, a measure of the strength of the attractions, increases by about $0.26-0.27 k_{\mathrm{B}} T$ when exchanging hydrogen for deuterium in the solvent. This proves that the isotope content of the solvent indeed has a dramatic effect on the protein interactions inducing a shift in critical temperature $T_{\mathrm{c}}$ of $16 \mathrm{~K}$ between pure $\mathrm{H}_{2} \mathrm{O}$ and pure $\mathrm{D}_{2} \mathrm{O}$. This value is noticeably larger than the shift reported in the transition (or denaturation) temperature $T_{\mathrm{m}}$ of globular proteins, which is $2-6 \mathrm{~K}$ when $\mathrm{D}_{2} \mathrm{O}$ is substituted for $\mathrm{H}_{2} \mathrm{O}$ in the solvent. ${ }^{10,13}$ Gripon et al. ${ }^{24}$ reported a shift in the value of the second virial coefficient, as well as in the solubility of lysozyme in $\mathrm{H}_{2} \mathrm{O}$ compared to $\mathrm{D}_{2} \mathrm{O}$, corresponding to about $7.2 \mathrm{~K}$. This value is equal to the difference between the temperature of maximum density of $\mathrm{H}_{2} \mathrm{O}$ and $\mathrm{D}_{2} \mathrm{O}$, again considerably smaller than the value found in our study. It has also been shown that near ambient temperature, $\mathrm{H}_{2} \mathrm{O}$ has a slightly more disordered structure than $\mathrm{D}_{2} \mathrm{O}$. The difference between $\mathrm{H}_{2} \mathrm{O}$ and $\mathrm{D}_{2} \mathrm{O}$ structure at a given temperature corresponds to the temperature-induced variation of either the $\mathrm{H}_{2} \mathrm{O}$ or $\mathrm{D}_{2} \mathrm{O}$ structure by a temperature shift of 5-10 K. ${ }^{38,39}$ Furthermore, at lower temperatures, quantum effects become more pronounced. ${ }^{38,39}$ Combined with the fact that hydrogen bonds in $\mathrm{D}_{2} \mathrm{O}$ are expected to be stronger than in $\mathrm{H}_{2} \mathrm{O}$ by $0.1-0.2 \mathrm{kcal} \mathrm{mol}^{-140}$ and that their number generally increases with lowering temperature, this leads us to speculate that an improved understanding of the quantum mechanical nature of the hydrogen bond in a biologically relevant temperature regime $(270-310 \mathrm{~K})$ may help to elucidate the effects of heavy water on protein folding 
and phase behavior, as well as the toxicity of $\mathrm{D}_{2} \mathrm{O}$ and its potential as an anticancer agent. The mechanisms behind this large effect of solvent isotope content on the critical point for liquid-liquid phase separation of $\gamma \mathrm{B}$-crystallin reported here are still not fully understood at this moment. Further studies into the underlying causes, as well as to establish whether this is a generic phenomenon also applying to other globular proteins, are thus clearly necessary.

\section{EXPERIMENTAL METHODS}

Following the method described by Thurston, ${ }^{41} \gamma \mathrm{B}$-crystallin was isolated and purified from fresh calf lenses, purchased from a local slaughterhouse. Final solvents with different $\mathrm{H}_{2} \mathrm{O} / \mathrm{D}_{2} \mathrm{O}$ mixing ratios were used in this study (cf. Table 1 ).

SLS experiments were performed on either a home-built multiangle light scattering instrument ${ }^{42}$ or on a commercial goniometer system (3D LS Spectrometer from LS Instruments $\left.\mathrm{AG}^{43}\right)$. SLS covers a range of scattering vectors $q=[4 \pi n(\sin \theta /$ $2)] / \lambda$ from 0.001 to $0.003 \AA^{-1}$, with $\theta$ being the scattering angle, $n$ the refractive index of the solvent, and $\lambda$ the wavelength of the radiation.

Refractive index measurements were performed on an Abbe refractometer at three different $\lambda$ and extrapolated to the wavelength under consideration.

$B_{2}$ values were determined by SLS on dilute solutions using the commercial goniometer instrument at a scattering angle $\theta=$ $90^{\circ}$.

In order to access $q$ values much larger than those covered by SLS, we utilized the SAXS technique. Measurements were performed on a Ganesha 300 XL SAXS System from SAXSLAB with an accessible $q$ range of $0.003-2.5 \AA^{-1}$. Data was corrected for background radiation, transmission, solvent, and capillary and was normalized with the protein concentration. The $c$ normalized scattering intensity is expressed as $I(q) / c \sim P(q)$ $S(q)$, where $S(q)$ is the static structure factor and $P(q) \sim I_{0}(q)$ / $c_{0}$ is the form factor with $P(q \rightarrow 0)=1$. $I_{0}(q)$ is the measured intensity of a dilute solution (where $S(q) \approx 1$ ) with concentration $c_{0}$. Experimentally, the structure factors of concentrated samples are then given by $S(q)=[I(q) / c] /$ $\left[I_{0}(q) / c_{0}\right]$.

\section{ASSOCIATED CONTENT}

\section{S Supporting Information}

The Supporting Information is available free of charge on the ACS Publications website at DOI: 10.1021/acs.jpclett.6b00593.

Details of sample preparation. Details of binodal, spinodal, and second virial coefficient $B_{2}$ determination. 3D plot of full data set of Figure $4 a$ and $b$. (PDF)

\section{AUTHOR INFORMATION}

\section{Corresponding Authors}

*E-mail: Najet.Mahmoudi@fkem1.lu.se.

*E-mail: Anna.Stradner@fkem1.lu.se.

\section{Present Addresses}

(L.C.-D.) Aventure AB, SE-223 63 Lund, Sweden.

(M.J.) Department of Applied Mathematics, Research School of Physical Sciences and Engineering, Australian National University, Canberra, Australian Capital Territory 2601, Australia.

(C.J.) Agroscope, Institute for Livestock Sciences ILS, CH1725 Posieux, Switzerland.

\section{Author Contributions}

(S.B and N.M.) These authors contributed equally to this work. Notes

The authors declare no competing financial interest.

\section{ACKNOWLEDGMENTS}

The authors thank Yuki Umehara for her help with the protein purification and sample preparation and Prof. Dr. Stefan Egelhaaf for helpful discussions. We gratefully acknowledge financial support from the Swiss National Science Foundation (SNF, grant 200020-127192), the Swedish Research Council (VR, grants 621-2012-2422 and 2009-6794), the Crafoord foundation (grants 20120619 and 20140756), and the Knut and Alice Wallenberg Foundation (project grant KAW 2014.0052).

\section{REFERENCES}

(1) Lewis, G. N.; Macdonald, R. T. Concentration of $\mathrm{H}^{2}$ Isotope. J. Chem. Phys. 1933, 1, 341-344.

(2) Kushner, D. J.; Baker, A.; Dunstall, T. G. Pharmacological Uses and Perspectives of Heavy Water and Deuterated Compounds. Can. J. Physiol. Pharmacol. 1999, 77, 79-88.

(3) Kumar, N.; Attri, P.; Yadav, D. K.; Choi, J.; Choi, E. H.; Uhm, H. S. Induced Apoptosis in Melanocytes Cancer Cell and Oxidation in Biomolecules Through Deuterium Oxide Generated from Atmospheric Pressure Non-Thermal Plasma Jet. Sci. Rep. 2014, 4, 7589.

(4) Ikeda, M.; Suzuki, S.; Kishio, M.; Hirono, M.; Sugiyama, T.; Matsuura, J.; Suzuki, T.; Sota, T.; Allen, C. N.; Konishi, S.; et al. Hydrogen-Deuterium Exchange Effects on $\beta$-Endorphin Release from AtT20 Murine Pituitary Tumor Cells. Biophys. J. 2004, 86, 565-575.

(5) Januschowski, K.; Mueller, S.; Spitzer, M.; Schramm, C.; Doycheva, D.; Bartz-Schmidt, K.-U.; Szurman, P. Evaluating Retinal Toxicity of a New Heavy Intraocular Dye, Using a Model of Perfused and Isolated Retinal Cultures of Bovine and Human Origin. Graefe's Arch. Clin. Exp. Ophthalmol. 2012, 250, 1013-1022.

(6) Antonino, L. C.; Kautz, R. A.; Nakano, T.; Fox, R. O.; Fink, A. L. Cold Denaturation and ${ }^{2} \mathrm{H}_{2} \mathrm{O}$ Stabilization of a Staphylococcal Nuclease Mutant. Proc. Natl. Acad. Sci. U. S. A. 1991, 88, 7715-7718.

(7) Guzzi, R.; Sportelli, L.; La Rosa, C.; Milardi, D.; Grasso, D. Solvent Isotope Effects on Azurin Thermal Unfolding. J. Phys. Chem. B 1998, 102, 1021-1028.

(8) Huyghues-Despointes, B. M. P.; Scholtz, J. M.; Pace, C. N. Protein Conformational Stabilities Can Be Determined from Hydrogen Exchange Rates. Nat. Struct. Biol. 1999, 6, 910-912.

(9) Cho, Y.; Sagle, L. B.; Iimura, S.; Zhang, Y.; Kherb, J.; Chilkoti, A.; Scholtz, J. M.; Cremer, P. S. Hydrogen Bonding of $\beta$-Turn Structure Is Stabilized in $\mathrm{D}_{2}$ O. J. Am. Chem. Soc. 2009, 131, 15188-15193.

(10) Makhatadze, G. I.; Clore, G. M.; Gronenborn, A. M. Solvent Isotope Effect and Protein Stability. Nat. Struct. Biol. 1995, 2, 852855.

(11) Cioni, P.; Strambini, G. B. Effect of Heavy Water on Protein Flexibility. Biophys. J. 2002, 82, 3246-3253.

(12) Parker, M. J.; Clarke, A. R. Amide Backbone and Water-Related H/D Isotope Effects on the Dynamics of a Protein Folding Reaction. Biochemistry 1997, 36, 5786-5794.

(13) Efimova, Y. M.; Haemers, S.; Wierczinski, B.; Norde, W.; Well, A. A. v. Stability of Globular Proteins in $\mathrm{H}_{2} \mathrm{O}$ and $\mathrm{D}_{2} \mathrm{O}$. Biopolymers 2007, 85, 264-273.

(14) Fu, L.; Villette, S.; Petoud, S.; Fernandez-Alonso, F.; Saboungi, M.-L. H/D Isotope Effects in Protein Thermal Denaturation: The Case of Bovine Serum Albumin. J. Phys. Chem. B 2011, 115, 18811888.

(15) Pelton, J. T.; McLean, L. R. Spectroscopic Methods for Analysis of Protein Secondary Structure. Anal. Biochem. 2000, 277, 167-176.

(16) Barth, A. Infrared Spectroscopy of Proteins. Biochim. Biophys. Acta, Bioenerg. 2007, 1767, 1073-1101. 
(17) Gabel, F.; Bicout, D.; Lehnert, U.; Tehei, M.; Weik, M.; Zaccai, G. Protein Dynamics Studied by Neutron Scattering. Q. Rev. Biophys. 2002, 35, 327-367.

(18) Bu, Z.; Biehl, R.; Monkenbusch, M.; Richter, D.; Callaway, D. J. E. Coupled Protein Domain Motion in Taq Polymerase Revealed by Neutron Spin-echo Spectroscopy. Proc. Natl. Acad. Sci. U. S. A. 2005, 102, 17646-17651.

(19) Schurtenberger, P. In Neutrons, X-Rays and Light: Scattering Methods Applied to Soft Condensed Matter; Lindner, P., Zemb, T., Eds.; Elsevier: Amsterdam, 2002; Chapter 7 (Contrast and Contrast Variation in Neutron, X-Ray and Light Scattering).

(20) Pancoska, P.; Wang, L.; Keiderling, T. A. Frequency Analysis of Infrared Absorption and Vibrational Circular Dichroism of Proteins in $\mathrm{D}_{2} \mathrm{O}$ Solution. Protein Sci. 1993, 2, 411-419.

(21) Cavanagh, J.; Fairbrother, W. J.; Palmer, A. G. I.; Rance, M.; Skelton, N. J. Protein NMR Spectroscopy: Principles and Practice, 2nd ed.; Elsevier/Academic Press: Amsterdam, 2007; Chapter 3 (Experimental Aspects of NMR Spectroscopy), pp 114-270.

(22) Gripon, C.; Legrand, L.; Rosenman, I.; Vidal, O.; Robert, M. C.; Boué, F. Lysozyme Solubility in $\mathrm{H}_{2} \mathrm{O}$ and $\mathrm{D}_{2} \mathrm{O}$ Solutions: A Simple Relationship. J. Cryst. Growth 1997, 177, 238-247.

(23) Broutin, I.; Riès-Kautt, M.; Ducruix, A. Lysozyme Solubility in $\mathrm{H}_{2} \mathrm{O}$ and $\mathrm{D}_{2} \mathrm{O}$ Solutions as a Function of Sodium Chloride Concentration. J. Appl. Crystallogr. 1995, 28, 614-617.

(24) Gripon, C.; Legrand, L.; Rosenman, I.; Vidal, O.; Robert, M.; Boué, F. Lysozyme-Lysozyme Interactions in Under- and SuperSaturated Solutions: A Simple Relation Between the Second Virial Coefficients in $\mathrm{H}_{2} \mathrm{O}$ and $\mathrm{D}_{2} \mathrm{o}$. J. Cryst. Growth 1997, 178, 575-584.

(25) Stradner, A.; Cardinaux, F.; Schurtenberger, P. Comment on "Effective Long-Range Attraction between Protein Molecules in Solution Studied by Small Angle Neutron Scattering”. Phys. Rev. Lett. 2006, 96, 219801.

(26) Stradner, A.; Thurston, G. M.; Schurtenberger, P. Tuning ShortRange Attractions in Protein Solutions: From Attractive Glasses to Equilibrium Clusters. J. Phys.: Condens. Matter 2005, 17, S2805S2816.

(27) Thomson, J. A.; Schurtenberger, P.; Thurston, G. M.; Benedek, G. B. Binary Liquid Phase Separation and Critical Phenomena in a Protein/Water Solution. Proc. Natl. Acad. Sci. U. S. A. 1987, 84, 70797083.

(28) Schurtenberger, P.; Chamberlin, R. A.; Thurston, G. M.; Thomson, J. A.; Benedek, G. B. Observation of Critical Phenomena in a Protein-Water Solution. Phys. Rev. Lett. 1989, 63, 2064-2067.

(29) Bucciarelli, S.; Casal-Dujat, L.; De Michele, C.; Sciortino, F.; Dhont, J.; Bergenholtz, J.; Farago, B.; Schurtenberger, P.; Stradner, A. Unusual Dynamics of Concentration Fluctuations in Solutions of Weakly Attractive Globular Proteins. J. Phys. Chem. Lett. 2015, 6, 4470-4474.

(30) Cardinaux, F.; Gibaud, T.; Stradner, A.; Schurtenberger, P. Interplay between Spinodal Decomposition and Glass Formation in Proteins Exhibiting Short-Range Attractions. Phys. Rev. Lett. 2007, 99, 118301.

(31) Noro, M. G.; Frenkel, D. Extended Corresponding-States Behavior for Particles with Variable Range Attractions. J. Chem. Phys. 2000, 113, 2941-2944.

(32) Gibaud, T.; Cardinaux, F.; Bergenholtz, J.; Stradner, A.; Schurtenberger, P. Phase Separation and Dynamical Arrest for Particles Interacting with Mixed Potentials - the Case of Globular Proteins Revisited. Soft Matter 2011, 7, 857-860.

(33) Platten, F.; Valadez-Pérez, N. E.; Castaneda-Priego, R.; Egelhaaf, S. U. Extended Law of Corresponding States for Protein Solutions. J. Chem. Phys. 2015, 142, 174905.

(34) Biffi, S.; Cerbino, R.; Bomboi, F.; Paraboschi, E. M.; Asselta, R.; Sciortino, F.; Bellini, T. Phase Behavior and Critical Activated Dynamics of Limited-Valence DNA Nanostars. Proc. Natl. Acad. Sci. U. S. A. 2013, 110, 15633-15637.

(35) Kleinert, H. Critical Exponents from Seven-Loop StrongCoupling $\varphi^{4}$ Theory in Three Dimensions. Phys. Rev. D: Part. Fields 1999, 60, 085001.
(36) Pelissetto, A.; Vicari, E. Critical Phenomena and Renormalization-Group Theory. Phys. Rep. 2002, 368, 549-727.

(37) Lu, P. J.; Zaccarelli, E.; Ciulla, F.; Schofield, A. B.; Sciortino, F.; Weitz, D. A. Gelation of Particles with Short-Range Attraction. Nature 2008, 453, 499-503.

(38) Hart, R. T.; Mei, Q.; Benmore, C. J.; Neuefeind, J. C.; Turner, J. F. C.; Dolgos, M.; Tomberli, B.; Egelstaff, P. A. Isotope Quantum Effects in Water Around the Freezing Point. J. Chem. Phys. 2006, 124, 134505.

(39) Bergmann, U.; Nordlund, D.; Wernet, P.; Odelius, M.; Pettersson, L. G. M.; Nilsson, A. Isotope Effects in Liquid Water Probed by X-Ray Raman Spectroscopy. Phys. Rev. B: Condens. Matter Mater. Phys. 2007, 76, 024202.

(40) Scheiner, S.; Cuma, M. Relative Stability of Hydrogen and Deuterium Bonds. J. Am. Chem. Soc. 1996, 118, 1511-1521.

(41) Thurston, G. M. Liquid-Liquid Phase Separation and Static Light Scattering of Concentrated Ternary Mixtures of Bovine $\alpha$ - and rB-Crystallins. J. Chem. Phys. 2006, 124, 134909-10.

(42) Moitzi, C.; Vavrin, R.; Bhat, S. K.; Stradner, A.; Schurtenberger, P. A New Instrument for Time-Resolved Static and Dynamic LightScattering Experiments in Turbid Media. J. Colloid Interface Sci. 2009, $336,565-574$.

(43) Urban, C.; Schurtenberger, P. Characterization of Turbid Colloidal Suspensions Using Light Scattering Techniques Combined with Cross-Correlation Methods. J. Colloid Interface Sci. 1998, 207, $150-158$. 